\title{
Neutrino Phenomenology
}

\author{
Pilar Coloma* \\ Fermi National Accelerator Laboratory \\ P.O. Box 500, Batavia 60510 IL, USA \\ E-mail: pcoloma@fnal.gov
}

\begin{abstract}
Neutrino oscillations have demonstrated that neutrinos have mass and, by now, oscillation experiments have been able to determine most of the parameters in the leptonic mixing matrix with a very good accuracy. Nevertheless, there are still many open questions in the neutrino sector. I will briefly discuss some of these questions, pointing out possible experimental avenues to address them.
\end{abstract}

XIII International Conference on Heavy Quarks and Leptons

22- 27 May, 2016

Blacksburg, Virginia, USA

${ }^{*}$ Speaker. 


\section{Open questions in neutrino physics}

The discovery of neutrino oscillations has opened many questions which still remain unanswered. The addition of neutrino masses to the Standard Model (SM) lagrangian is not a straightforward task to begin with, as new particles are needed. For this purpose, perhaps the most naive way to extend the SM could be through the addition of right-handed neutrinos $v_{R}$ to its particle content. This would allow for a Yukawa term with the Higgs $\phi$ and the left-handed doublets $L_{L}$, in the form $Y_{v} \bar{L}_{L} \tilde{\phi} v_{R}$. After electroweak symmetry breaking (EWSB) this term would generate Dirac masses for the neutrinos, just as it is done for the rest of the SM fermions.

However, in order to produce neutrino masses below the eV scale through this mechanism, a Yukawa coupling $Y_{v} \sim 10^{-12}$ would be needed. Even though this is perfectly viable, it poses interesting questions regarding the naturalness of such a small coupling especially since the ones associated to their doublet counterparts, the charged leptons, are at least six orders of magnitude larger. Furthermore, a right-handed neutrino is a singlet under the SM gauge group and, thus, once it is included in the SM nothing forbids the addition of a Majorana mass term in the form of $M v_{R}^{c} v_{R}$ to the lagrangian, except the conservation of lepton number. This brings up the question of whether lepton number is a fundamental symmetry of the SM or just an accidental one. Does the new physics violate lepton number explicitly? Furthermore, what is the scale of new physics associated to this new mass $M$ ? If lepton number is violated explicitly, additional signatures might be observable in future experiments. Neutrinoless double beta decay processes are a well-known example to conduct these searches, but lepton number violation might be observable as well in rare meson decays or even in collider experiments.

Since the discovery of neutrino oscillations, the progress in the field has been outstanding. The three angles involved in the leptonic mixing matrix are now known within a precision which ranges between approximately $8 \%$ for the atmospheric mixing angle $\theta_{23}$ and a $3 \%$ for the reactor angle $\theta_{13}$ [1]. Two very different squared mass splittings have also been measured with a very good accuracy: these show that at least two of the light neutrino mass eigenstates are massive, while the third eigenstate could in principle be massless. Such outstanding effort in neutrino oscillation experiments has revealed that the observed pattern in the mixing matrix is very different from the one measured in the quark sector, leading to the so-called "flavor puzzle": why are these mixing patterns so different? Is there a flavor symmetry behind the observed structure that explains the values of these mixing angles?

Models which advocate for the existence of flavor symmetries try to address these questions, but they will not be able to prosper unless the experimental community provides precise measurements for all the relevant parameters which govern flavor mixing in the SM extended with neutrino masses. The fact that the atmospheric mixing angle lies very close to maximal mixing makes it extremely difficult to determine whether it is exactly maximal or not and, if not maximal, the octant it belongs to. Furthermore, even though we know that the three angles in the leptonic mixing matrix are non-zero, currently there is only a hint for the value of the Dirac CP-violating phase. The ordering of neutrino masses (i.e., whether the lightest mass eigenstate is $v_{1}$ or $v_{3}$ ) remains also unknown. Current and future long-baseline experiments aim to provide answers to these three questions, which have important consequences in model building for neutrino masses and mixing. 


\section{Lepton number violating signatures}

For concreteness, let us focus on the naive scenario in which we extend the SM with three righthanded neutrinos $v_{R}$. As already explained, as lepton number is not part of the gauge symmetry of the SM, in this case one would have to consider both Yukawa and Majorana mass terms in the neutrino mass lagrangian:

$$
\mathscr{L}_{v} \supset Y_{v} \bar{L}_{L} \tilde{\phi} v_{R}+\frac{1}{2} M \bar{v}_{R}^{c} v_{R}+\text { h.c. }
$$

After the Higgs acquires a vacuum expectation value $v$, the full neutrino mass matrix in the basis $\left(v_{L}, v_{R}^{c}\right)$ reads:

$$
\mathscr{M}=\left(\begin{array}{cc}
0 & Y_{v} v \\
Y_{v}^{\dagger} v & M
\end{array}\right) .
$$

The limit when $M \gg Y_{v} v$ is particularly appealing. In this limit, after diagonalizing the mass matrix in Eq. 2.2, the eigenvalues take the form

$$
\begin{aligned}
m_{i} & \sim v^{2} Y_{v}^{\dagger} M^{-1} Y_{v}, & (i=1,2,3), \\
m_{i} & \sim M+\mathscr{O}\left(v^{2} Y^{\dagger} M^{-1} Y\right) & (i=4,5,6),
\end{aligned}
$$

where $m_{i}$ and $M_{i}$ refer to the masses of the three eigenstates in the light sector and the heavy sector. As can be seen from Eq. 2.3, the smallness of the light neutrino masses is explained through the suppression with a scale of new physics much higher than the EW scale, without the need for very small Yukawa couplings. This is the well-known See-Saw Type I mechanism [2, 3].

However, a priori there is no strong reason which forces the Majorana masses to lie above the EW scale. In particular, the limit when $M \rightarrow 0$ is also interesting, as in this limit the number of symmetries in the lagrangian increases (lepton number is recovered). Furthermore, if the scale of new physics $M$ is below the EW scale, additional phenomenological consequences could take place at low energies. For example, for $M \sim \mathscr{O}(\mathrm{eV})$, new states would be present at the eV-scale: this could offer an explanation for the observed anomalies in short-baseline experiments. On the other hand, a right-handed neutrino at the $\mathrm{keV}$ scale would be a good candidate for warm dark matter. Higher scales could have interesting phenomenological consequences as well: for example, the existence of additional neutrinos in the $\mathrm{MeV}-\mathrm{GeV}$ regime could lead to lepton number violating signatures in rare meson decays or in beta decays.

The addition of relatively light right-handed neutrinos could have an impact in lepton flavor violating processes as well. Currently, the strongest experimental constraints impose the impressive upper bound on the branching ratio for $\mu \rightarrow e \gamma, B(\mu \rightarrow e \gamma)<4.2 \times 10^{-13}$ [4]. In presence of right handed neutrinos, an extra contribution to the branching ratio for this process would be generated. This imposes tight constraints on the mixing between light and heavy states, as the extra contribution goes like $[5,6,7]$

$$
B(\mu \rightarrow e \gamma)=\frac{3 \alpha}{8 \pi}\left|\sum_{I} V_{e I} V_{\mu I}^{*} f\left(\frac{m_{I}^{2}}{m_{W}^{2}}\right)\right|^{2}
$$

Here, the sum on $I$ runs only over heavy mass eigenstates, $\alpha$ is the electroweak fine constant, $m_{W}$ is the mass of the $W$ boson, and $f(x)$ is a loop function which takes values between 0 and 1 as $x$ 
goes from 0 to infinity. It should be noted that the elements of the mixing matrix $V$ which mix the light and heavy sectors are roughly proportional to the ratio between the light and heavy masses.

Finally, the existence of additional light neutrinos could also lead to observable deviations from the expected observable rate for neutrinoless-double beta decay. In this case, the situation is a bit more complicated, as the unitarity of the whole mixing matrix leads to exact cancellations when all the new mass eigenstates lie either below or above the energy scale associated to the nuclear transition $(\mathscr{O}(100) \mathrm{MeV})$, see e.g. Ref. [8, 9] for a detailed discussion. However, in scenarios where there is a large hierarchy between the Majorana masses associated to the right-handed neutrinos, such cancellations could in principle be avoided $[9,10]$. This may lead to a considerably different rate with respect to the expected result in the standard scenario, where only the light neutrinos participate in the process. For instance, in Ref. [11] a phenomenological study was performed including a sterile neutrino at the $\mathrm{eV}$ scale. This example highlights the importance of cross checks of different measurements in order to pin down possible effects coming from new physics. For example, if long-baseline experiments determine that the neutrino mass ordering is normal, and the neutrinoless double beta decay rate is measured to be compatible with the inverted ordering scenario, this could be pointing out to the existence of new physics which violates lepton number at low energies, see, e.g. Ref. [12].

\section{Oscillations, $\mathrm{CP}$ violation and the flavor puzzle}

The current and future generation of neutrino oscillation experiments that will aim at determining the mass ordering can be divided into three main categories: long-baseline neutrino oscillation, atmospheric neutrino and medium-baseline reactor experiments. A quantitative comparison between the expected performance of these facilities and their projected sensitivities to the mass ordering can be found, e.g., in Ref. [13]. Both long-baseline (T2K [14], NOvA [15], DUNE [16]) and atmospheric neutrino oscillation experiments (ICAL at INO [17], PINGU [18], ORCA [19], Hyper-Kamiokande [20]) are sensitive to the mass ordering through the observation of the MSW effect $[21,22]$. It leads to a resonant enhancement of the oscillation probabilities for normal ordering for neutrino oscillations, while in the inverted ordering scenario the resonance takes place for antineutrinos. On the other hand, reactor experiments at medium baselines (JUNO [23] and RENO-50 [24]) are sensitive to this observable through the appearance of an interference pattern in the oscillation probabilities in vacuum between the solar and the atmospheric contributions to the oscillation amplitude. The exact location of the interference and its evolution with the neutrino energy would be different depending on whether $\Delta m_{31}^{2}$ is larger or smaller than $\Delta m_{32}^{2}$, and thus probes the neutrino mass ordering directly.

However, the major goal for the current and future generation of long-baseline neutrino experiments is to determine whether there is CP violation in the lepton sector. In the SM there are two possible sources of $\mathrm{CP}$ violation: the $\mathrm{CP}$ phase involved in the $\mathrm{CKM}$ matrix, which has been measured to be very close to $\sim 70^{\circ}$, and the $\theta$-vacuum of QCD, which is experimentally bounded to be below $\theta<\mathscr{O}\left(10^{-10}\right)$ [25]. There is little understanding as to why these parameters take these values. Thus, determining whether there are additional $\mathrm{CP}$-violating sources in the lepton sector could help to shed light onto this long-standing problem of particle physics. 
Recent data from the T2K [26] and NOvA [27] experiments (in combination with a very precise determination of $\theta_{13}$ from reactor experiments) currently seem to point to a negative value of $\delta$, possibly maximally CP-violating. In the upcoming years, they will keep accumulating additional statistics which may strengthen this hint further. If $\delta$ is indeed close to $-90^{\circ}$, the sensitivity obtained from the combination of T2K and NOvA data could reach a significance of $\sim 2 \sigma$ for this observable (see, e.g., Ref. [28]). Their final combined sensitivity will eventually depend on other factors as well, such as the true values of $\theta_{23}$ and the neutrino mass ordering. Future experiments such as DUNE or T2HK will be much more sensitive to this observable, with an expected signal significance above $5 \sigma$ if $\delta$ is around $\pm 90^{\circ}$ and $\theta_{23}$ lies in the lower octant $[29,20]$.

The discovery of $\mathrm{CP}$ violation in the lepton sector would be a major step forward in particle physics. Nevertheless, a precise measurement of the value of $\delta$ would have important consequences for model building in the flavor sector as well. The flavor puzzle in the SM has been object of extensive investigations, especially since the discovery of the large mixing angles which dominate neutrino mixing: while the CKM mixing matrix is very close to being diagonal, the leptonic mixing matrix obeys a completely different pattern. Flavor models attempt to explain the large differences observed in the mixing between quarks and between leptons, sometimes even linking the two sectors. In this context, models based on discrete symmetries have become very popular. In some of these, tight relations between the observable mixing angles in the PMNS matrix and the CPviolating phase ( $a k a$, sum rules) take place. Therefore, a precise determination of all parameters in the PMNS matrix could allow to discriminate between them. In fact, current measurements of the three mixing angles already allow to reject some of these flavor models, as their corresponding sum rules provide predictions for $\cos \delta$ which lie in unphysical regions of the parameter space (see Fig. 3 in Ref. [30]). Additional recent works along these lines include Refs. [31, 32, 33, 34, 35, 36, 37], among others.

In order to further discriminate among different flavor models, it is therefore crucial to improve the determination of the mixing angles (in particular the atmospheric mixing angle, which currently holds the largest uncertainty) and, especially, to get a precise measurement of $\delta$. Current experiments will provide a hint on whether $\delta$ is CP-violating or not; however, if its value turns out to be close to $\delta \sim \pm 90^{\circ}$ (as favored by current data), T2K and NOvA will only determine its value with a rather large error bar, $\sim 70^{\circ}$ [38]. In other words, having a hint for CP-violation does not guarantee a precise measurement of the $\mathrm{CP}$-violating phase itself a priori. Again in this case, future experiments with larger statistics and better precision will be needed for this purpose. The final precision achievable for $\delta$ will depend on its value: DUNE is expected to reach an accuracy around $\sim 15^{\circ}$ at $1 \sigma$ [29]; a similar precision is expected for T2HK [20].

\section{Summary}

The discovery of neutrino masses has evidenced the existence of new particles beyond the SM. Even in the simplest scenario when only right-handed neutrinos are added to the SM particle content, new questions remain to be answered. If lepton number is just an accidental symmetry of the SM model, the inclusion of Majorana masses for the right-handed neutrinos seems mandatory: this implies the existence of a new physics scale. In this talk I have given a few examples of new observable phenomena which could take place in rare meson decays, colliders, lepton flavor 
violating observables and neutrinoless double beta decay, among others. The final answer will depend on the physics scale responsible for the generation of neutrino masses.

I have also stressed out the complementarity between different experimental searches and its importance in order to pin down possible effects coming from new physics in the neutrino sector. As an example, I discussed the importance of the determination of the neutrino mass ordering in order to interpret the results from neutrinoless double beta decay searches. Finally, I have also discussed the relevance of CP-violation searches in the lepton sector and, most importantly, the precise determination of the value of the CP-violating phase. This could provide valuable data to attack the flavor puzzle in the SM extended with neutrino masses.

Acknowledgments. I would like to warmly thank the organizers of the HQL conference for their invitation to give this talk, and the Physics Department at Virginia Tech for providing partial support to attend this conference. Fermilab is operated by Fermi Research Alliance, LLC under Contract No. DE-AC02-07CH11359 with the United States Department of Energy. This work was partially supported by the European Union's Horizon 2020 Research and Innovation Program under the Marie Sklodowska-Curie grant agreements No. 690575 and 674896.

\section{References}

[1] M. C. Gonzalez-Garcia, M. Maltoni and T. Schwetz, Updated fit to three neutrino mixing: status of leptonic CP violation, JHEP 1411, 052 (2014) doi:10.1007/JHEP11(2014)052 [arXiv:1409.5439 [hep-ph]].

[2] P. Minkowski, $\mu \rightarrow$ er at a Rate of One Out of $10^{9}$ Muon Decays?, Phys. Lett. B 67, 421 (1977). doi:10.1016/0370-2693(77)90435-X

[3] M. Gell-Mann, P. Ramond and R. Slansky, Complex Spinors and Unified Theories, Conf. Proc. C 790927, 315 (1979) [arXiv:1306.4669 [hep-th]].

[4] A. M. Baldini et al. [MEG Collaboration], Search for the Lepton Flavour Violating Decay $\mu^{+} \rightarrow e^{+} \gamma$ with the Full Dataset of the MEG Experiment, Eur. Phys. J. C 76, no. 8, 434 (2016) doi:10.1140/epjc/s10052-016-4271-x [arXiv:1605.05081 [hep-ex]].

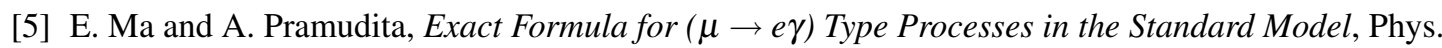
Rev. D 24, 1410 (1981). doi:10.1103/PhysRevD.24.1410

[6] P. Langacker and D. London, Lepton Number Violation and Massless Nonorthogonal Neutrinos, Phys. Rev. D 38, 907 (1988). doi:10.1103/PhysRevD.38.907

[7] D. Tommasini, G. Barenboim, J. Bernabeu and C. Jarlskog, Nondecoupling of heavy neutrinos and lepton flavor violation, Nucl. Phys. B 444, 451 (1995) doi:10.1016/0550-3213(95)00201-3 [hep-ph/9503228].

[8] M. Blennow, E. Fernandez-Martinez, J. Lopez-Pavon and J. Menendez, Neutrinoless double beta decay in seesaw models, JHEP 1007, 096 (2010) doi:10.1007/JHEP07(2010)096 [arXiv:1005.3240 [hep-ph]].

[9] J. Lopez-Pavon, S. Pascoli and C. f. Wong, Can heavy neutrinos dominate neutrinoless double beta decay?, Phys. Rev. D 87, no. 9, 093007 (2013) doi:10.1103/PhysRevD.87.093007 [arXiv:1209.5342 [hep-ph]]. 
[10] J. Lopez-Pavon, E. Molinaro and S. T. Petcov, Radiative Corrections to Light Neutrino Masses in Low Scale Type I Seesaw Scenarios and Neutrinoless Double Beta Decay, JHEP 1511, 030 (2015) doi:10.1007/JHEP11(2015)030 [arXiv:1506.05296 [hep-ph]].

[11] C. Giunti and E. M. Zavanin, Predictions for Neutrinoless Double-Beta Decay in the 3+1 Sterile Neutrino Scenario, JHEP 1507, 171 (2015) doi:10.1007/JHEP07(2015)171 [arXiv:1505.00978 [hep-ph]].

[12] A. Ibarra, E. Molinaro and S. T. Petcov, TeV Scale See-Saw Mechanisms of Neutrino Mass Generation, the Majorana Nature of the Heavy Singlet Neutrinos and $(\beta \beta)_{0 v}$-Decay, JHEP 1009, 108 (2010) doi:10.1007/JHEP09(2010)108 [arXiv:1007.2378 [hep-ph]].

[13] M. Blennow, P. Coloma, P. Huber and T. Schwetz, Quantifying the sensitivity of oscillation experiments to the neutrino mass ordering, JHEP 1403, 028 (2014) doi:10.1007/JHEP03(2014)028 [arXiv:1311.1822 [hep-ph]].

[14] Y. Itow et al. [T2K Collaboration], The JHF-Kamioka neutrino project, hep-ex/0106019.

[15] D. S. Ayres et al. [NOvA Collaboration], The NOvA Technical Design Report, FERMILAB-DESIGN-2007-01.

[16] R. Acciarri et al. [DUNE Collaboration], Long-Baseline Neutrino Facility (LBNF) and Deep Underground Neutrino Experiment (DUNE) : Volume 1: The LBNF and DUNE Projects, arXiv:1601.05471 [physics.ins-det].

[17] For details of the India-based Neutrino Observatory, visit www.ino.tifr.res.in/

[18] M. G. Aartsen et al. [IceCube Collaboration], PINGU: A Vision for Neutrino and Particle Physics at the South Pole, arXiv:1607.02671 [hep-ex].

[19] A. Kouchner, KM3NeT - ORCA: measuring the neutrino mass ordering in the Mediterranean, J. Phys. Conf. Ser. 718, no. 6, 062030 (2016). doi:10.1088/1742-6596/718/6/062030

[20] K. Abe et al., Letter of Intent: The Hyper-Kamiokande Experiment - Detector Design and Physics Potential —, arXiv:1109.3262 [hep-ex].

[21] L. Wolfenstein, Neutrino Oscillations in Matter, Phys. Rev. D 17, 2369 (1978). doi:10.1103/PhysRevD.17.2369

[22] S. P. Mikheev and A. Y. Smirnov, Resonance Amplification of Oscillations in Matter and Spectroscopy of Solar Neutrinos, Sov. J. Nucl. Phys. 42, 913 (1985) [Yad. Fiz. 42, 1441 (1985)].

[23] F. An et al. [JUNO Collaboration], Neutrino Physics with JUNO, J. Phys. G 43, no. 3, 030401 (2016) doi:10.1088/0954-3899/43/3/030401 [arXiv:1507.05613 [physics.ins-det]].

[24] S. B. Kim, New results from RENO and prospects with RENO-50, Nucl. Part. Phys. Proc. 265-266, 93 (2015) doi:10.1016/j.nuclphysbps.2015.06.024 [arXiv:1412.2199 [hep-ex]].

[25] K. A. Olive et al. [Particle Data Group Collaboration], Review of Particle Physics, Chin. Phys. C 38, 090001 (2014). doi:10.1088/1674-1137/38/9/090001

[26] K. Abe et al. [T2K Collaboration], Observation of Electron Neutrino Appearance in a Muon Neutrino Beam, Phys. Rev. Lett. 112, 061802 (2014) doi:10.1103/PhysRevLett.112.061802 [arXiv:1311.4750 [hep-ex]].

[27] P. Adamson et al. [NOvA Collaboration], First measurement of electron neutrino appearance in NOvA, Phys. Rev. Lett. 116, no. 15, 151806 (2016) doi:10.1103/PhysRevLett.116.151806 [arXiv:1601.05022 [hep-ex]]. 
[28] S. K. Agarwalla, S. Prakash, S. K. Raut and S. U. Sankar, Potential of optimized NOvA for large $\left.\theta_{(} 13\right)$ \& combined performance with a LArTPC \& T2K, JHEP 1212, 075 (2012) doi:10.1007/JHEP12(2012)075 [arXiv:1208.3644 [hep-ph]].

[29] R. Acciarri et al. [DUNE Collaboration], Long-Baseline Neutrino Facility (LBNF) and Deep Underground Neutrino Experiment (DUNE) : Volume 2: The Physics Program for DUNE at LBNF, arXiv:1512.06148 [physics.ins-det].

[30] P. Ballett, S. F. King, C. Luhn, S. Pascoli and M. A. Schmidt, Testing solar lepton mixing sum rules in neutrino oscillation experiments, JHEP 1412, 122 (2014) doi:10.1007/JHEP12(2014)122 [arXiv:1410.7573 [hep-ph]].

[31] P. Ballett, S. Pascoli and J. Turner, Mixing angle and phase correlations from A5 with generalized CP and their prospects for discovery, Phys. Rev. D 92, no. 9, 093008 (2015) doi:10.1103/PhysRevD.92.093008 [arXiv:1503.07543 [hep-ph]].

[32] A. Di Iura, C. Hagedorn and D. Meloni, Lepton mixing from the interplay of the alternating group $A_{5}$ and CP, JHEP 1508, 037 (2015) doi:10.1007/JHEP08(2015)037 [arXiv:1503.04140 [hep-ph]].

[33] I. Girardi, S. T. Petcov, A. J. Stuart and A. V. Titov, Leptonic Dirac CP Violation Predictions from Residual Discrete Symmetries, Nucl. Phys. B 902, 1 (2016) doi:10.1016/j.nuclphysb.2015.10.020 [arXiv:1509.02502 [hep-ph]].

[34] I. Girardi, S. T. Petcov and A. V. Titov, Predictions for the Leptonic Dirac CP Violation Phase: a Systematic Phenomenological Analysis, Eur. Phys. J. C 75, 345 (2015) doi:10.1140/epjc/s10052-015-3559-6 [arXiv:1504.00658 [hep-ph]].

[35] I. Girardi, S. T. Petcov and A. V. Titov, Determining the Dirac CP Violation Phase in the Neutrino Mixing Matrix from Sum Rules, Nucl. Phys. B 894, 733 (2015) doi:10.1016/j.nuclphysb.2015.03.026 [arXiv:1410.8056 [hep-ph]].

[36] P. Ballett, S. F. King, C. Luhn, S. Pascoli and M. A. Schmidt, Testing atmospheric mixing sum rules at precision neutrino facilities, Phys. Rev. D 89, no. 1, 016016 (2014) doi:10.1103/PhysRevD.89.016016 [arXiv:1308.4314 [hep-ph]].

[37] M. C. Chen, M. Fallbacher, Y. Omura, M. Ratz and C. Staudt, Predictivity of models with spontaneously broken non-Abelian discrete flavor symmetries, Nucl. Phys. B 873, 343 (2013) doi:10.1016/j.nuclphysb.2013.04.020 [arXiv:1302.5576 [hep-ph]].

[38] P. Coloma, A. Donini, E. Fernandez-Martinez and P. Hernandez, Precision on leptonic mixing parameters at future neutrino oscillation experiments, JHEP 1206, 073 (2012) doi:10.1007/JHEP06(2012)073 [arXiv:1203.5651 [hep-ph]]. 